\title{
Aprender Juntos a Vivir y Explorar la Complejidad. Nuevos Marcos Pedagógicos de Interpretación y Acción
}

\section{Learning Together to Live and Explore Complexity. New Pedagogical Frameworks}

\author{
Ángel I. Pérez Gómez *, Encarnación Soto Gómez \\ Universidad de Málaga, España
}

\begin{abstract}
DESCRIPTORES:
Sabiduría

Aprendizaje

Enseñanza

Evaluación

Lesson study

\section{RESUMEN:}

Este trabajo presenta un análisis y diagnóstico de las características globales y desafíos educativos que este año de pandemia ha subrayado como tareas pendientes de nuestro sistema educativo. Aprender a vivir en la incertidumbre, cultivar la sabiduría, promover el cuidado y la cooperación, fomentar la conciencia ecológica con un modelo económico al servicio de la vida y comprender, cuestionar e integrar educativamente los potentes escenarios virtuales y las controvertidas herramientas digitales, son para nosotros los retos ineludibles y urgentes que debemos abordar, para aprender juntos a vivir y explorar la complejidad. Responder de manera adecuada a estos retos requiere apostar decididamente, en la escuela y en la universidad, por nuevos marcos pedagógicos de comprensión y acción que incorporen una participación y una intervención más activa, reflexiva, cooperativa, personalizada, creativa e híbrida. En definitiva, necesitamos incorporar formas de enseñar y evaluar, que faciliten que cada aprendiz vaya construyendo y reconstruyendo en cooperación y con autonomía su propio proyecto vital y profesional. La Lesson Study, como estrategia de investigación acción cooperativa centrada en la mejora de la enseñanza desde la perspectiva de los estudiantes y el desarrollo profesional docente inicial y permanente, puede considerarse una valiosa estrategia pedagógica para este propósito.
\end{abstract}

\section{KEYWORDS:}

Wisdom

Learning

Teaching

Evaluation

Lesson study

\section{ABSTRACT:}

This paper presents an analysis and diagnosis of the global characteristics and educational challenges that this pandemic year has highlighted as pending tasks for our educational system. Learning to live in uncertainty, cultivating wisdom, promoting care and cooperation, fostering ecological awareness with an economic model at the service of life, and understanding, questioning and educationally integrating powerful virtual scenarios and controversial digital tools, are for us, the unavoidable and urgent challenges that we must address, in order to learn together to live and explore complexity. Responding appropriately to these challenges requires a firm commitment, at school and university, to new pedagogical frameworks of understanding and action that incorporate more active, reflective, cooperative, personalised, creative and hybrid participation and intervention. In short, we need to incorporate ways of teaching and assessing that enable each learner to build and rebuild their own life and professional project in cooperation and autonomy. The Lesson Study, as a cooperative action research strategy focused on the improvement of teaching from the students' perspective and the initial and ongoing professional development of teachers, can be considered a valuable pedagogical strategy for this purpose.

CÓMO CITAR:

Pérez Gómez, A. I. y Soto Gómez, E. (2021). Aprender juntos a vivir y explorar la complejidad. Nuevos marcos pedagógicos de interpretación y acción. REICE. Revista Iberoamericana sobre Calidad, Eficacia y Cambio en Educación, 19(4), 13-29.

https://doi.org/10.15366/reice2021.19.4.001

*Contacto: apgomez@uma.es

ISSN: 1696-4713

revistas.uam.es/reice 


\section{Los desafíos educativos de una época de pandemias}

Son innumerables las aportaciones y reflexiones compartidas sobre los fenómenos vividos en este largo y excepcional año de emergencia sanitaria mundial, sus causas, desarrollos y consecuencias, en relación con los desafíos sociales, políticos, profesionales y educativos que emergen. Para el propósito de esta reflexión nos permitimos resumir de forma breve, aquellos retos educativos que nos parecen más críticos y relevantes para atender la terrible, extraña e incierta era pandémica planetaria que no ha dejado ningún área de la vida sin tocar, sin remover. Una pedagogía responsable y respondiente no puede dejar de enfrentar con urgencia y decisión los retos actuales que, sin ser nuevos, reaparecen de forma intensa y se tornan ineludibles, demandando un cambio sustancial de prioridades para la teoría y sobre todo para la práctica pedagógica. Destacaremos los siguientes.

\subsection{Aprender a vivir en la incertidumbre y manejar la fragilidad y el cambio permanente. Vulnerabilidad y resiliencia}

Esta era de complejidad, incertidumbre y cambio acelerado requiere seres humanos cada vez más flexibles, reflexivos y empáticos; mejor preparados para indagar, pensar y actuar de manera cooperativa en escenarios tan inciertos y complejos.

A este respecto, nos parece clave repensar el termino vulnerabilidad, como una condición sustantiva del ser humano, que implica reconocer la fragilidad, el carácter efímero, el error y el no saber, tanto como la apertura, el deseo y la posibilidad ilimitada de búsqueda, cambio y creación, ante las situaciones habituales o excepcionales que habita (Braintmeier y Mackenna, 2020). La extraordinaria plasticidad neuronal ${ }^{1}$ del cerebro sustenta esta doble y paradójica característica del ser humano cuyos dos polos aparentemente incompatibles, es necesario considerar y atender como tendencias complementarias de un mismo devenir complejo, tortuoso y enriquecedor. Brown (2016) considera que la vulnerabilidad no es debilidad, es la esencia de todas las emociones y sentimientos, el corazón, el centro de las experiencias humanas significativas. Crecer es aceptar la vulnerabilidad. Estar vivo es ser vulnerable.

El resultado de aceptar una idea respetuosa y recíproca de la vulnerabilidad humana es abrir las posibilidades a la necesidad de vínculo, de confianza y compromiso con los demás. Salir de nuestro aislamiento, nuestra coraza defensiva y atreverse, arriesgarse a ser vulnerables con otras personas. Cuando nos pasamos la vida, consciente o inconscientemente, a la defensiva, tratando de evitar la vulnerabilidad, no nos queda espacio para la incertidumbre, el riesgo, la exposición y apertura emocional, el error, el aprendizaje y la creación. Nuestra vida emocional abierta y libre implica necesariamente aceptar la vulnerabilidad, la incertidumbre del caminar humano que descubre nuevos horizontes en un proceso inacabado de reinvención y cambio individual y social (Louden, 2020). Reconocer la vulnerabilidad como constitutiva del ser humano nos abre el camino a la búsqueda resiliente de las experiencias, escenarios y relaciones más adecuados para construir el bienestar personal y colectivo. Admitir la ignorancia, compartir y coaprender pueden surgir como las estrategias privilegiadas para compaginar la vulnerabilidad y la resiliencia, proyectando al ser humano a afrontar de forma decidida el riesgo de vivir. El conocimiento y el no saber, el egoísmo y el altruismo, no necesariamente constituyen una dicotomía incompatible, una relación de combate, sino una polaridad en tensión, una alianza generativa (Challenger, 2021; Garcés, 2020). Buscar el equilibrio entre tendencias divergentes no supone la mediocridad y banalidad del término medio, requiere vivir con intensidad aceptando las contradicciones y las compensaciones de los polos alternativos que constituyen nuestra existencia, afrontar el saludable contraste de ideas, sin ceder a la tentación de enfrentar de manera agresiva posiciones y posturas vitales polarizadas e irreconciliables. Entender la complejidad paradójica de esta interacción es un requisito, imprescindible para elaborar nuestras descripciones, explicaciones y propuestas pedagógicas

La complejidad humana ${ }^{2}$ y su inseparable incertidumbre se ubica tanto en la enmarañada red de relaciones económicas, técnicas, políticas y culturales que constituyen el cada día más espinoso y sofisticado

1 Para comprender la riqueza de este concepto nos parece interesante recordar la distinción que propone Garcés (2020): Mientras que la flexibilidad se define únicamente como la capacidad de recibir una o múltiples formas, la plasticidad incluye también la de crear, modificar e incluso destruir toda forma adquirida.

2 Cabe recordar a este respecto la distinción que Innerarity (2020), citando a Luhmann (1997), establece entre 
mundo -mundos- que vivimos (Harari, 2018, 2020; Innerarity, 2020; Sennet, 2018), como en la complicada y peculiar estructura de nuestros propios mecanismos personales cognitivos y afectivos, conscientes y subconscientes, de comprensión, toma de decisiones y actuación (Barrett, 2018; Fuster, 2020; Kahneman 2015; Pugh, 2019; Sawyer 2019). Estos dos polos, externo e interno, en permanente interacción y tensión, constituyen la complejidad vulnerable y la potencialidad de lo que consideramos el sujeto humano, como individuo y como colectivo. Como complejos sistemas vivos, los seres humanos solo sobreviviremos si, sabiéndonos seres inacabados, abiertos, frágiles y vulnerables somos capaces de aprender, de adaptarnos a un contexto en permanente cambio, perpetua novedad. ${ }^{3}$

\subsection{Cultivar la sabiduría. La honestidad existencial}

Cultivar la sabiduría, requiere transitar de la información al pensamiento crítico, práctico y creativo, alimentando la coherencia entre el decir, el pensar y el hacer. En esta era de supercomplejidad, la carencia de información no es el problema. El problema se sitúa en la calidad de dicha información, así como en la capacidad de utilizar el mejor conocimiento disponible para generar experiencias de bienestar individual y social, precisamente en un contexto enredado en la manipulación, la sobreabundancia, la charlatanería, la postverdad. Para afrontar de manera satisfactoria la vulnerabilidad constitutiva del ser humano en un mundo extraordinariamente complejo y cambiante no sirve cualquier tipo de conocimiento. El reto es construir conocimiento informado, complejo, experiencial y útil, individual y colectivo, consciente y subconsciente, para poder entender los múltiples hilos, procesos y matices que conlleva la complejidad y ser capaces de situarnos e intervenir en ella (Pérez Gómez, 2017).

Cuando la vida está en juego empieza a palidecer el truco de las apariencias, la promoción del simulacro y la impostura, y se impone priorizar lo esencial, la relevancia y calidad del saber. El aprendizaje de informaciones para presumir o reproducir en exámenes deja de tener sentido. El valor de uso del conocimiento sustituye al conocimiento con valor de cambio por notas, calificaciones u otros intereses espurios. Lo esencial y relevante prima sobre lo accidental y oportunista, el aprendizaje profundo sobre el aprendizaje superficial, la ciencia y el arte sobre la palabrería, el diseño y la planificación reflexiva sobre el beneficio a corto plazo, los atajos y los engaños.

El concepto de sabiduría, utilizar el mejor conocimiento para el gobierno de la propia vida como individuos o colectivos, se relaciona de manera estrecha con el concepto de honestidad existencial, e implica necesariamente la integración coherente de las formulaciones teóricas y las aplicaciones prácticas. La enseñanza educativa no puede conformarse con formulaciones retóricas, ha de procurar la coherencia y honestidad entre las teorías proclamadas, las practicas promovidas y las experiencias vivenciadas, de modo que los valores que defendemos a veces alegremente, a veces de manera hipócrita, se conviertan en principios de procedimiento, en virtudes, en estrategias de acción e indagación y en modos de vivir y de hacer. Sabemos mucho más de lo que aplicamos y predicamos mucho más y mejor de lo que hacemos. Asumir las contradicciones y limitaciones humanas no excusa, ni justifica la hipocresía pedagógica, por el contrario, anima y estimula el esfuerzo por superarlas y manejarlas de manera realista, honesta y consciente. Innerarity (2020) plantea de manera lúcida e insistente la imperiosa necesidad contemporánea de aprender a manejarnos con un saber incompleto, que no arbitrario, y reaccionar adecuadamente ante lo inesperado. En sentido similar, Sawyer (2019) nos recuerda acertadamente que la información y el conocimiento en si no es tan relevante, porque es efímero, incremental, inabarcable por cada individuo y está en google actualizado y contrastado. Lo verdaderamente relevante es qué haces con el mejor conocimiento para abordar los problemas complejos y cambiantes de la sociedad contemporánea, qué nuevas posibilidades se generan, qué innovación se activa. En el qué hacer con el conocimiento influye tanto la calidad del conocimiento como las habilidades, las actitudes, la motivación y las disposiciones subjetivas

complejidad y complicación. La complicación se refiere a la estructura, mientras que la complejidad se fija en la dinámica, pues lo decisivo es el comportamiento cambiante de los elementos. Una estructura puede ser complicada pero no compleja porque su mecánica lineal no produce emergencia de procesos imprevisibles.

3 A este respecto, Barad (2007) desarrolla el sugerente concepto de intracción. Parte del convencimiento de que las entidades reales y en particular los sistemas vivos no son cosas separadas que entran en relación, sino elementos que se construyen desde el principio en virtud de las relaciones y contextos que habitan. Los individuos no preexisten a sus interacciones, son el producto de tales intrarelaciones entrelazadas. 
conscientes y no conscientes, para manejarlo, individual y colectivamente. Por ello, cultivar la sabiduría individual o colectiva es mucho más que adquirir y reproducir información y conocimiento declarativo, requiere cualidades y prácticas cognitivas y socioemocionales de nivel superior.

\subsection{Promover de manera decidida y urgente el cuidado y la cooperación}

Afirmar la centralidad de los sentimientos altruistas en la construcción compartida de propósitos comunes. Tomar conciencia de la constitutiva interdependencia y fragilidad planetaria de los seres humanos evidencia la necesidad de ayudarnos en este devenir sorprendente e incierto. La cooperación no puede ser ya una estrategia de supervivencia de cada grupo o tribu local, sino una manera universal de entender las relaciones humanas, micro y macro. El cuidado del otro, la atención solidaria y la compasión aparecen como las actitudes y desafíos humanos más urgentes y necesarios para afrontar la fragilidad, para facilitar la convivencia saludable, la supervivencia sostenible y el florecer de la dignidad humana. Paradójicamente, las profesiones peor pagadas en la actualidad son las más imprescindibles en tiempos de pandemia, crisis y emergencia. Se necesita un nuevo contrato social que sitúe como prioridad la convivencia solidaria, que proteja la vida sostenible y saludable y que se preocupe no solo de proclamar los derechos humanos sino de practicarlos, de hacerlos viables en los modos de organización económica, social y política, tanto como en la construcción, desde la infancia, de los hábitos subjetivos correspondientes. La profundización de la democracia como filosofía y forma práctica de vida. Si la responsabilidad de este nuevo ethos cultural del cuidado convoca a toda la ciudadanía, la escuela, la universidad y los docentes somos los profesionales más directamente apelados y comprometidos en su desarrollo cotidiano, conscientes de que la reciprocidad y el aprecio mutuo es la relación clave que vincula a los seres humanos, que aceptan su vulnerabilidad e interdependencia, su no saber y sus ilimitadas posibilidades de crear, de aprender, desaprender y reaprender.

Como ha puesto en evidencia esta etapa de pandemia, desarrollar y practicar el cuidado mutuo no constituye un lujo ni una opción; es una necesidad básica para el bienestar de los individuos y la supervivencia de la especie. Ahora bien, el desarrollo de la cooperación y del resto de habilidades sociales no es un proceso sencillo, ni automático. Es cierto que como afirma la investigación en antropología y ciencias sociales, la selección natural nos ha equipado con una extraordinaria sensibilidad social, con capacidad de amor, amistad, aprendizaje y cooperación (Christakis, 2019). Pero al mismo tiempo, ese mismo proceso evolutivo nos ha equipado para buscar la supervivencia, en primer lugar, del propio individuo y del propio grupo, la tribu, reaccionando a la defensiva con desconfianza y agresividad ante la presencia de extraños, o ante quien, supuesta o realmente, la amenace. Somos capaces, por tanto, de lo mejor y de lo peor. Por ello, aprender a desarrollar habilidades, actitudes, creencias y sentimientos que favorezcan la cooperación, el cuidado, la compasión y el apoyo mutuo es la responsabilidad irrenunciable de cada generación y requiere, un largo, intencionado y complejo proceso, que apela a los individuos y a las instituciones desde los primeros momentos de la vida. El bienestar de nuestra especie y de nuestro planeta depende del desarrollo de nuestra inteligencia social y emocional en favor de la compasión y del cuidado mutuo.

\subsection{Fomentar la conciencia ecológica y promover un modelo económico al servicio de la vida es para nosotros el cuarto desafío urgente}

La pandemia ha puesto de manifiesto el fracaso del capitalismo, en especial en su despiadada versión ultraliberal, para resolver los problemas serios que asolan a los ciudadanos (Escolar, 2020). Hemos comprobado en estos meses que este modelo económico parece derrumbarse cuando solo consumimos lo necesario, pero no cuando el beneficio superlativo de unos pocos provoca la miseria de muchos. El beneficio financiero privado a cualquier precio, por encima de la satisfacción de necesidades reales de la humanidad y de la sostenibilidad del medio natural, la desregulación, la privatización, la deslocalización, la sobreexplotación de los recursos naturales y la desforestación conducen al incremento obsceno de la desigualdad y al desastre ecológico. Supone, por tanto, una evidente y seria amenaza de la propia supervivencia de la especie humana, (Garret, 2020). ¿Alguien puede entender que, en una era tan científica y técnicamente desarrollada, la población mundial se encuentre indefensa porque los productos y servicios imprescindibles, -personal médico, hospitales, mascarillas, respiradores, UCI y vacunas-, dependan de empresas, corporaciones y oligopolios privados? Se requiere, por tanto, promover otro modelo que persiga de manera decidida la primacía del bien común y el cuidado de las personas y del medio natural. 
Attali (2020), propone sustituir con urgencia la economía del libre mercado por la economía de la vida, centrada en la atención y el cuidado de las personas, donde los sectores prioritarios tienen que ver con el cuidado de los otros y del medio natural. Salud, educación, higiene, medio ambiente, agricultura, alimentación, cultura digital y servicios sociales, que se perfilan como las áreas prioritarias de la economía de la vida, deben situarse en el espacio de lo común, de lo público, al margen de la lucha por el beneficio privado. Necesitamos superar las desigualdades de todo tipo, si queremos construir una convivencia auténticamente democrática, tan amenazada por las tendencias ultraliberales e iliberales y las derivas autoritarias, supremacistas e insolidarias actuales. De modo muy evidente y crudo, la pandemia sanitaria ha puesto de manifiesto la necesidad de recuperar y fortalecer las instituciones y servicios públicos sobre los mercados privados. Solo, una apuesta decidida y valiente por una economía de la vida, a favor de la equidad y la sobriedad, puede revertir esta grave crisis de confianza social.

En un mundo global e inevitablemente interdependiente se requiere avanzar hacia la gobernanza democrática mundial para enfrentar los problemas globales y responder a la interdependencia universal. La declaración universal de los derechos humano supuso en su día un paso sustancial en esta dirección, pero la deriva neoliberal de finales de los 80 del siglo pasado y la versión ultraliberal más reciente han provocado la ruptura de aquella tímida e inicial tendencia hacia la gobernanza mundial democrática (Innerarity, 2020). La escuela debe abrazar este reto como uno de los más relevantes y urgentes de su quehacer, pues la verdadera democracia es una filosofía de vida que debe adquirirse en la práctica, desde las primeras interacciones que conforman nuestros modos habituales de percibir, interpretar, tomar decisiones y actuar.

\subsection{Comprender, cuestionar e integrar educativamente los potentes escenarios virtua- les y las poderosas y controvertidas herramientas digitales}

La prolongada cuarentena vivida, a la que probablemente tengamos que acostumbrarnos, ha obligado a hacer una transición digital de urgencia que ha puesto de manifiesto tanto la relevancia de la enseñanza virtual, como la magnitud de la brecha digital, las tendencias tóxicas que pueden vehicular las redes sociales, así como las insuficiencias estructurales y las deficiencias en la preparación de los docentes para trabajar en dichos escenarios (Soloway y Norris, 2020).

$\mathrm{Ni}$ apocalípticos ni integrados, la tecnología digital se impone de manera irreversible en la vida cotidiana del ciudadano contemporáneo, condicionando sus modos de percibir, pensar, comunicar y actuar. Cómo afirma Ramonet (2020), Google, Amazon, Facebook o Netflix, son las triunfadoras absolutas, en términos económicos, de este momento trágico de la historia. El regreso triunfal de los oligopolios (Rivas, 2020; Vidal, 2020,). Vivimos la época de los Big data, la nueva materia prima, dominante en la era de pandemias. La biovigilancia justificada por necesidades sanitarias, el teletrabajo, el telecomercio, el teleconsumo, así como la teleenseñanza y la telesanidad se proponen como los formatos de relaciones humanas privilegiados en esta época de crisis y obligado distanciamiento, acelerando, sin el reposo, el debate y la reflexión necesarias, la implantación de una manera controvertida de entender la era digital (Eagleman, 2020; Seymur, 2020; Veliz, 2020; Zuboff, 2020). En todo caso, parece incuestionable que el territorio virtual es tan real como el presencial y cada día ocupa más tiempo de la vida de los humanos.

La sabiduría, como la mejor manera de gobernarse a sí mismo, se complica a medida que vivimos en escenarios virtuales y presenciales cada vez más complejos, interconectados, inciertos y cambiantes. Mención especial merecen a este respecto los sorprendentes desarrollos de la Inteligencia Artificial (IA) en la última década (Zuboff, 2020). En principio, la IA no tiene por qué sustituir o desplazar a las personas, ha de considerarse como un complemento excelente, una extensión artificial de sus capacidades de percibir, interpretar, tomar decisiones y actuar. Pero los desarrollos actuales se mueven siempre en el filo de la autonomía, planteando y cuestionando el papel del ser humano en su control y supervisión. Por otra parte, es urgente afrontar las consecuencias inevitables que conlleva la automatización en la vida cotidiana. Desaparecerán muchos yacimientos laborales y se abrirán otros muchos, pero de índole bien diferente, pues requerirán capacidades cognitivas, afectivas y morales de nivel superior para poder diseñar, desarrollar, evaluar y controlar la interacción con los artefactos inteligentes. El reto será proponer y crear nuevas responsabilidades y trabajos que los humanos puedan hacer mejor que un algoritmo, tareas que 
impliquen creatividad, pensamiento interdisciplinar, interacción y atención humana, gestión y cuidado de las emociones, juicios de valor y compromiso moral, así como el decisivo territorio, precisamente, del diseño, desarrollo, control y supervisión de estas poderosas máquinas inteligentes. Es imprescindible y urgente crear conciencia, empoderar a la ciudadanía y defender con ahínco el derecho a la intimidad frente al vendaval de los big data (Torralba,2020; Veliz, 2020).

Stephen Hawkings ya alertó hace años del riesgo que para la raza humana supone el avance en inteligencia artificial de compañías privadas sin control, ni supervisión. No podemos olvidar que desde el punto de vista educativo la tecnología es un excelente siervo, pero también un terrible amo, pues puede amenazar la deseada autodeterminación humana. Su complejidad y sofisticación es de tal naturaleza que los expertos no son capaces de explicar a veces el porqué y el cómo (O’Neil, 2018; Vidal, 2018). Se abren desarrollos autónomos de las máquinas, que desbordan la capacidad de comprensión de la mayoría de los ciudadanos, dificultando con ello el verdadero control democrático y responsable.

Por otra parte, no podemos sucumbir al pesimismo y a la desesperanza. Parece obligado destacar también el lado positivo de las tecnologías contemporáneas. Como propone Eagleman (2020), a diferencia de pandemias pasadas, en la presente, precisamente por el elevado desarrollo de las tecnologías digitales, hemos sabido y podido construir una poderosa red universal de comunicación digital, que nos permite relacionarnos, vernos, sentir, comprar, producir y trabajar desde casa, así como, investigar, experimentar y producir vacunas, estar informados, tomar decisiones colectivas y democráticas, sin peligro alguno de contaminación. Sin embargo, es imprescindible interrogarnos si nosotros controlaremos la IA o si la IA, en manos de corporaciones multinacionales privadas que ostenten el monopolio, nos controlará a nosotros, convirtiéndonos en Cibors (Veliz, 2020). La IA requiere una regulación global, una gobernanza mundial democrática que proteja la libertad y la autonomía de los seres humanos.

\section{Nuevos marcos pedagógicos. Enseñanza y evaluación basadas en la experiencia, reflexión, cooperación y creatividad}

Responder de manera adecuada a estos retos requiere nuevos marcos pedagógicos de interpretación y acción, apostar decididamente por una manera de enseñar y evaluar activa, reflexiva, cooperativa, personalizada y creativa. Un modo de enseñar y evaluar que facilite aprender juntos a explorar la complejidad, a construir en cooperación el proyecto vital propio, ensayando, experimentando, descubriendo los problemas reales del contexto, en permanente interacción con los miembros de su comunidad. ¿Será posible que la pandemia como acontecimiento crítico global genere la oportunidad de cambiar sustancialmente los modos de aprender, enseñar y evaluar en esta escuela ya obsoleta? (Pozo, 2020).

La escuela convencional, por lo general, de talla única, inspirada en los propósitos de la Ilustración y conformada por las exigencias de la industrialización, vigente hasta nuestros días, con independencia de sus múltiples méritos históricos y honrosas excepciones, no está diseñada para responder a la magnitud de estos desafíos. En la mayoría de sus prácticas, prima la transmisión directa, la memorización sin interés y a menudo sin sentido para el aprendiz; desmotiva, aburre y discrimina a quien no encaja la talla única que impone; descontextualiza, fomenta el aprendizaje superficial, aísla a los docentes, organiza de manera inflexible a los estudiantes por edades, en horarios y espacios rígidos, exige aprender de memoria un currículum organizado en disciplinas, de kilómetros de extensión y milímetros de profundidad, confunde los medios con los fines, tiene una idea muy restringida del conocimiento y de la inteligencia, relega la dimensión práctica y creativa del saber, ignora o desprecia dimensiones fundamentales y decisivas de la personalidad como las actitudes, los hábitos, las emociones y los valores, se ahoga obsesionada con la calificación/acreditación y olvida la evaluación educativa (Brady, 2020; Claxton y Lucas, 2015; Pérez Gómez, 2012, 2017; Pozo, 2020).

Es cierto que, en el último medio siglo, se ha avanzado de manera muy significativa en el desarrollo de un discurso pedagógico potente y fundamentado, pero, salvando valiosas y minoritarias excepciones, nuestras prácticas docentes siguen las inercias de esta obsoleta escuela heredada. Por ello, será imprescindible y urgente repensar de manera radical la teoría y la práctica del currículum, la enseñanza, la evaluación y la propia escuela como institución, y promover una nueva cultura pedagógica para cultivar la sabiduría crítica y práctica y potenciar decididamente la estrategia de aprender juntos a arriesgarnos a 
vivir la vulnerabilidad y promover la resiliencia en tiempos de complejidad e incertidumbre. Todos los componentes esenciales del complejo sistema de enseñanza aprendizaje que tiene lugar en las instituciones académicas requieren un cambio sustancial para asumir la responsabilidad de preparar a los futuros ciudadanos para comprender y vivir la complejidad técnica, política, económica, social y cultural del escenario contemporáneo. Nosotros, aquí, vamos a focalizar solamente algunos aspectos que se relacionan con la metodología de enseñanza y evaluación. En este monográfico pueden encontrarse desarrollos más exhaustivos relacionados con el resto de componentes: currículum, profesorado, organización institucional, políticas educativas...

Por su relevancia pedagógica nos detendremos en cinco ejes básicos que agrupan los principios didácticos que, a nuestro entender, fundamentan la renovación sustancial de la institución escolar. Desarrollaremos los principios, los marcos $\mathrm{y}^{4}$ la filosofía pedagógica de unos modos de hacer coherentes con los retos y los propósitos anteriormente expuestos, cuyos múltiples modos didácticos de derivación en estrategias y técnicas concretas deben quedar abiertos a la experimentación situacional de cada escenario educativo y de sus agentes responsables. Concluiremos el texto con la exposición, a modo de un ejemplo, de las propuestas que nosotros venimos desarrollando durante la última década en la formación inicial y permanente del profesorado, en la universidad de Málaga.

\subsection{Promover la experiencia educativa relevante y reflexiva}

El territorio educativo se mueve en este espacio complejo y paradójico, en el que nos construimos como sujetos relativamente autónomos, cuestionando y reformulando, a la luz del mejor conocimiento disponible, la validez antropológica de los influjos que hemos recibido e incorporado en nuestro largo y permanente proceso de socialización cotidiana ${ }^{5}$. Educarse supone, por tanto, reconstruir no solamente los modelos mentales conscientes y explícitos, las teorías declaradas, sino de manera muy especial los patrones, hábitos, creencias y mapas mentales subconscientes y tácitos que vamos construyendo en virtud de las experiencias concretas que gobiernan nuestros deseos, inclinaciones, interpretaciones, decisiones y reacciones automáticas. Esta reconstrucción consciente del piloto automático no es un propósito simple, requiere tanto la acción, como la contemplación, el contraste y la reflexión sobre el sentido y la eficacia de nuestra acción. Se requiere desarrollar cualidades cognitivas y socioemocionales de nivel superior, un pensamiento práctico informado, autónomo y creativo. Es decir, el desarrollo singular y holístico en cada individuo de las dimensiones sustanciales de su personalidad, las cualidades, capacidades o competencias como sistemas complejos de comprensión, autoorganización y actuación, que incluyen al mismo nivel y con la misma relevancia, conocimientos, habilidades, emociones, actitudes y valores como sus componentes esenciales (Korthagen, 2017; Pérez Gómez, 2012, 2017; Soto et al., 2015).

Estos recursos de comprensión y acción se adquieren y se reconstruyen en sucesivos procesos de aprendizaje experiencial profundo ${ }^{6}$, mediante la reflexión en y sobre la acción. Las experiencias educativas requieren sumergirse en el flujo abierto, incierto e incluso contradictorio de la singular, compleja y cambiante experiencia vivida. Percibir y comprender la integración precaria, en un inestable equilibrio entre caos y rigidez potenciando a la vez, la diferenciación y el vínculo (Siegel, 2017). La acción educativa no se identifica con el activismo o la hiperactividad, requiere autenticidad, vincularse con problemas auténticos en contextos reales, presenciales o virtuales, un abordaje plural que incluya la contemplación, la acción y la reflexión, el conocimiento público declarado y el omnipresente precocinado subconsciente singular de cada sujeto. La diferenciación, requiere relaciones intersubjetivas, contrastes informados y reflexiones metacognitivas; tanto tiempos y espacios de actividad y experimentación con sentido, como tiempos lentos de estudio y pausas existenciales (Poyton, 2020), de silencio y sosiego que permitan e inspiren la contemplación, la meditación y la reflexión para que cada aprendiz pueda asumir, a su propio ritmo, la responsabilidad de removerse y autoorganizarse con honestidad, buscando su propio equilibrio,

\footnotetext{
4 Utilizamos el concepto de marco desarrollado por Lakoff (2016), como estructuras mentales, fundamentalmente tácitas y subconscientes, que moldean nuestra visión del mundo, los objetivos que perseguimos, los planes que trazamos, el modo en que actuamos y lo que valoramos.

5 Un desarrollo más elaborado de la complejidad de la construcción educativa del sujeto humano puede encontrarse en Pérez Gómez $(2012,2017,2020)$.
}

6 Puede encontrarse un desarrollo más detenido del concepto de aprendizaje profundo en Pérez Gómez (2019). 
su propio proyecto, la compleja y frágil coherencia entre sus deseos, sueños, pensamientos, sentimientos, discursos y prácticas.

La responsabilidad didáctica de los docentes reside en la búsqueda de las condiciones pedagógicas que favorezcan y estimulen el desarrollo de estas experiencias educativas de todos y cada uno de los aprendices. Condiciones de espacio, tiempo, clima de relaciones, actividades y procesos que faciliten el desarrollo personalizado y el encuentro confiado y entusiasta de los aprendices en la exploración compartida de los misterios inacabables de la compleja realidad, en cambio permanente. Es obvio que esta forma de enseñar y evaluar requiere más tiempo, más dedicación, mayor y mejor preparación y un incremento sustancial del número de docentes.

Retomando la seminal propuesta de Stenhouse de hace más de 50 años, en aras de la coherencia pedagógica es imprescindible convertir los valores y supuestos que, como consecuencia de la deliberación democrática, consideramos pedagógicamente deseables, en principios de procedimiento que fundamenten las múltiples y diversificadas formas técnicas que pueden adoptar nuestras concretas y situacionales propuestas didácticas, (aprendizaje como investigación y experimentación: aprendizaje basado en problemas, proyectos, aprendizaje basado en el diseño, la investigación y la experimentación, ambientes de aprendizaje, Lesson Study, Aprendizaje y Servicio, Reggio Emilia approach,...) y de evaluación (evaluación como y para el aprendizaje, portfolios, diarios, relatos narrativos, procesos de análisis y diagnóstico de situaciones, casos y problemas...etc.). Nuestra responsabilidad es favorecer contextos y procesos autónomos y acompañados que potencien dicha coherencia y honestidad, pero no solo en los conocimientos declarativos, sino de manera muy especial en su dimensión existencial: emociones, actitudes, hábitos, creencias y valores.

El estudio, el aprendizaje memorístico de apuntes, e incluso el manejo correcto de conocimientos declarativos aislados y fragmentados para el éxito en los exámenes y pruebas de acreditación, no puede ser por más tiempo el marco, la piedra angular de nuestra pedagogía académica. Todo el progreso educativo hasta la universidad ha de promover experiencias educativas, es decir, experiencias de aprendizaje que faciliten e inspiren la reconstrucción permanente de los modos conscientes y subconscientes de percibir, interpretar, proponer, tomar decisiones, actuar y valorar. El cultivo de la sabiduría como pensamiento práctico, crítico y creativo. La utilización del mejor conocimiento disponible para el gobierno de nuestra compleja y paradójica vida personal, social y profesional (Pérez Gómez, 2017).

\subsection{Potenciar la colaboración y el compromiso social}

Vivir la vulnerabilidad y la resiliencia como propósitos pedagógicos sustanciales requiere promover en las instituciones escolares, de manera decidida y omnipresente la cooperación educativa. Aprender juntos a sumergirse en el complejo, incierto y misterioso rio de la vida a partir de la conciencia de lo que sabemos y de lo que no sabemos y de la necesidad de apoyarnos, cuidarnos y potenciarnos mutuamente como la mejor estrategia para vivir la grandeza y la miseria de la vulnerabilidad individual y colectiva. A este respecto me parece muy sugerente la propuesta de Garcés (2020) de promover la alianza de aprendices, aprendo contigo si tu aprendes conmigo, para sumergirse juntos en la aventura de conocer y aprender, como proceso de cocreacción. Potenciar experiencias compartidas de aprendizaje que incluyan interdependencia y diferenciación, personalización y cooperación, crear espacios de aprendizaje transformativos y emocionales, mediante la reciprocidad, la receptividad y una actitud de cuidado mutuo, para adentrarnos en el respetable y apasionante misterio de los retos de la vida (Brown, 2016; Noddings, 2018). La cooperación como encuentro de aprendices valora tanto las diferencias y el respeto a las discrepancias como el vínculo, así como en el convencimiento de que el todo, los todos, es mucho más que la suma de las partes.

Desde el punto de vista educativo, cuidar es acompañar, atender, responder a necesidades, pero, sobre todo, empoderar, favorecer que los estudiantes aprendan juntos a enfrentarse por sí mismos y en colaboración a los inciertos e inesperados vaivenes de la vida, (Torralba, 2020). Por eso, la cooperación no es una técnica más sino una filosofía pedagógica, el alma máter del modo de proceder humano como especie eminentemente social que aprende a celebrar la diversidad, consciente, en la actualidad, de que la cooperación solidaria debe extenderse no solo al grupo tribal próximo, sino a todas las culturas y sociedades que pueblan el planeta. Como ya se recoge, por ejemplo, en UBUNTU, una regla ética tradicional sudafricana que apela a la lealtad de las personas y las relaciones entre éstas, o en las propuestas japonesas de la pedagogía SOKA: el bien común es el bien propio y el bien propio se protege en el bien común. 
Este principio didáctico parece más necesario que nunca en la actualidad por la enorme dificultad de su realización. Porque es claramente contraintuitivo y contracultural en una atmosfera económica, política, social y cultural que ensalza el individualismo del sálvese quien pueda, el obsesivo e innecesario consumo privado que agota los recursos naturales, la competitividad salvaje que conduce al enfrentamiento hostil y a la polarización agresiva. Como propone Torralba (2020), precisamente ahora, para neutralizar el pesimismo ambiental en este panorama dantesco, la escuela deberá ser un pequeño pulmón de esperanza, un frágil ecosistema de reforestación del alma. Es urgente atreverse a construir ya en la escuela, en un diálogo permanente con los demás y con la naturaleza, un espacio para explorar y experimentar la vida que deseamos. Una vida que a veces acaricia y a veces sacude, que provoca, inspira, frustra e ilusiona, pero que siempre puede ser mucho más que la mera supervivencia, si nos atrevemos a convivir, compartir la presencia, con honestidad y curiosidad.

Nuestra responsabilidad como docentes se concreta de manera prioritaria en el diseño y desarrollo compartido con los aprendices de escenarios de convivencia donde puedan aprender por experiencia, que la confianza, el diálogo, el apoyo mutuo, el cariño y la colaboración de y entre todos suponen la clave de un aprendizaje más rico y profundo y sobre todo más satisfactorio para todos y para la naturaleza. Escenarios socioeducativos, donde aprender a valorar lo común por encima del ego propio, el terreno de lo público que a todos nos ampara sin invadir el terreno de lo privado y de lo íntimo, aprender a vivir y convivir en grupos sociales cada vez más heterogéneos, celebrando la diversidad y respetando las discrepancias, aprendiendo a erradicar la violencia física y psicológica, así como a resolver de manera pacífica, dialogada y democrática los inevitables conflictos que se generan en las comunidades humanas (Belavi y Murillo, 2020). Cómo afirma Morín en su reciente publicación (2020) arropar un yo dentro de un nosotros local y universal un Yonos como identidad compartida.

\subsection{Trabajar la improvisación guiada y compartida. Creatividad y asombro}

Entre los modos de hacer didáctico más adecuados a esta nueva cultura pedagógica se encuentra, a nuestro entender, la estrategia de potenciar la improvisación disciplinada, guiada, compartida, que supone cuidar el delicado equilibrio entre la libertad de la creación y el rigor del proceso de búsqueda y experimentación cooperativa. La improvisación guiada y compartida potencia la capacidad y el deseo de arriesgarse a descubrir sin límites, de adentrarse, en el territorio de lo desconocido, lo posible y lo inesperado, arropados, pero no limitados, por el conocimiento ya disponible (Sawyer, 2017).

Promover el desarrollo del pensamiento práctico, crítico y creativo, la sabiduría, requiere concebir y practicar la enseñanza como proceso de creación. No una creación desde la nada como en la mitología bíblica, sino concebida como encuentro, como la manera de traer a la presencia las existencias de otros, como hospitalidad, acogida de existencias múltiples y diferentes que se reinventan al interactuar, generando vínculos con la naturaleza, con los demás y con nosotros mismos. Una práctica poética que no solo produce obras, sino que crea sensibilidad (Garcés, 2020).

La creatividad no es una cualidad innata, ni reservada a una élite de genios, ni de personas excéntricas, es la capacidad de generar vínculos y relaciones de forma libre, es la práctica de hacer presente lo ausente. No es una expresión en el vacío sino asentada en el conocimiento teórico, empírico y experiencial, un hermoso y laborioso proceso de búsquedas compartidas, arriesgadas, sin miedo al error, que cuestiona lo ya adquirido abriéndose al misterio de los mundos posibles, planteando de manera permanente la duda, la búsqueda de matices y alternativas no explorados, que todos los seres humanos necesitamos a lo largo de nuestra vida personal, profesional y social ${ }^{7}$.

La creatividad se estimula y alimenta en contextos abiertos, donde reina la confianza y el estímulo a la iniciativa personal y al intercambio enriquecedor de diferentes formas de mirar, entender y expresar, donde el error se considera una ocasión de aprendizaje y donde se promueven proyectos retadores y complejos en los que los aprendices tienen que ensayar y proponer alternativas. Este espíritu de la indagación creativa, compartido y promovido en comunidades de investigación (Sawyer, 2017, 2019) es el que se precipita en lo que denominamos improvisación disciplinada, (científica, artística y poética), excelen-

7 A este respecto, Willson (2018) considera con acierto que los científicos más competentes son aquellos que piensan como poetas y trabajan como contables. 
temente ejemplificada en las sesiones libres de jazz (Sternberg et al., 2009). La docencia educativa en la era contemporánea de la complejidad, cambio e incertidumbre contiene un importantísimo componente creativo en cooperación, bien fundamentado, donde los agentes creadores improvisan juntos, resuenan juntos, comparten sentimientos, conocimientos, hábitos, técnicas, ritmos y patrones y dentro de ese marco compartido y sensibles a los diferentes contextos y aprendices, se mueven libremente dando rienda suelta, en sintonía, a la expresión singular de sus hipótesis, emociones, sentimientos y sueños, conectados al misterio que supone la búsqueda de la belleza, la bondad, el saber.

\subsection{Potenciar y reformular la enseñanza híbrida}

Otra variable decisiva de una pedagogía responsable ante los desafíos actuales es la consideración de los novedosos contextos pedagógicos digitales sobrevenidos. No podemos desaprovechar la oportunidad de comprender, criticar, y disfrutar educativamente la potencia de los escenarios virtuales analizando a fondo sus luces y sus sombras. La etapa de confinamiento no solo ha puesto frente al abismo al sistema, sino también a nuestra preparación como docentes. Necesitamos prepararnos para que el vértigo y la incertidumbre no nos paralice e impida descubrir, no solo las peligrosas derivas de las TIC sino también sus enormes fortalezas. La enseñanza a distancia no tiene por qué ser distante. Así lo ponen de manifiesto diferentes e interesantes proyectos y experiencias promovidos en lugares, instituciones y organismos diversos que han intentado atender esta difícil e incierta situación. ${ }^{8}$

Es cierto que la brusca e imprevista traslación de la escuela al hogar durante el confinamiento, supuso cambios sustanciales que situaron a los docentes en un terreno pantanoso de ambigüedad, desconocimiento, inexperiencia e incertidumbre, sobre qué y cómo enseñar, acompañar y evaluar el aprendizaje de los estudiantes en sus respectivos domicilios (Luengo y Manso, 2020; Trujillo, 2020) y a las familias ante un escenario complejo de conciliación y cuidado sobre todo en las edades tempranas. Pero la enseñanza educativa, no puede obviar la potencia del omnipresente escenario virtual, sino que ha de preparar para que los recursos, procesos, agrupamientos, redes, escenarios y materiales virtuales contribuyan al desarrollo más autónomo y holístico de la personalidad de todos y cada uno de los ciudadanos. En la era digital, aprender en casa puede y debe ser una continuación natural del aprendizaje en el aula y aprender en el aula ha de incorporar de manera crítica y creativa los procesos de aprendizaje vivenciados en la casa y en las interacciones sociales. La escuela educativa ha de prepararnos para afrontar contextos en los que la robotización, los big data, los algoritmos y la inteligencia artificial contribuyan al desarrollo óptimo y saludable de cada individuo y de la comunidad (Gewertz, 2020). Una enseñanza híbrida en la que el escenario virtual y presencial se potencien mutuamente para promover un mismo proyecto pedagógico.

Conscientes de la brecha social, cultural y digital entre las diferentes familias es necesario destacar los siguientes aspectos:

La acumulación perversa de los efectos de la confluencia de las diversas brechas, que impiden la igualdad de oportunidades, se intensifica y agranda en el escenario virtual, tanto por la diferente disponibilidad de recursos tecnológicos, espaciales y sociales, como por la enorme diferencia de apoyos humanos y familiares a los procesos educativos en los distintos hogares.

Es necesario redefinir el currículum y reformular la pedagogía híbrida; romper la estricta separación entre la escuela y el hogar de modo que un mismo proyecto pedagógico se desarrolle en diferentes escenarios, ambientes y formatos, atendiendo a las posibilidades y limitaciones de cada uno de ellos; promover la enseñanza personalizada; fomentar el trabajo cooperativo online tanto para tareas de enseñanza como de evaluación entre pares; esencializar el currículum, bajo el supuesto de que menos es más; fomentar la creación de espacios virtuales compartidos, sugerentes y flexibles; facilitar la complementariedad de tareas síncronas y asíncronas; aprovechar las posibilidades del aula invertida, pues complementa las inmejorables condiciones de transmisión de información rigurosa, actualizada, atractiva y persistente que ofrece la comunicación audiovisual asíncrona, con la riqueza de los intercambios, cercanos, ágiles,

8 Pueden consultarse al respecto: el proyecto del Ministerio de Educación de China: "Suspender las clases sin parar el aprendizaje ni la enseñanza" (Zhang et al., 2020); las recomendaciones desarrolladas por UNICEF para "reimaginar la educación” (Belmonte et al., 2020); las sugerentes historias compartidas por la Fundación Teach for All en su programa \#DontStopLearning; las prometedoras experiencias de Mitra (2019); las fundamentadas propuestas de Sangrá (2020), Allen y otros (2020), Hu y otros (2019), y Reimers y otros (2020). 
multisensoriales e interactivos que ofrece la modalidad presencial en mayor medida que la virtual, para dialogar, debatir, proyectar y evaluar en cooperación. En definitiva, solo integrando las TIC en el aula podremos mejorar su utilización y ayudar a los aprendices a hacer un uso más crítico, autónomo y reflexivo de los espacios digitales que habitan (Kaiser, 2019).

La tarea de recrear la propuesta pedagógica y adaptarla a las circunstancias tan diversas de los aprendices y sus contextos familiares desborda la disponibilidad y la capacidad actual de los docentes individualmente considerados y requiere refuerzos y cualidades especiales de preparación y actuación cooperativa, de todos los agentes que intervienen (Konen, 2020). Será, por tanto, necesario desarrollar estrategias de comunicación ágil y cercana con las familias y una disminución de las ratios para que puedan ejercer la esencial labor complementaria de acompañar el aprendizaje de los estudiantes que el formato virtual intensifica.

La motivación es la clave del éxito de la enseñanza en general y de forma muy especial de la enseñanza online, por cuanto supone mayor fuerza de voluntad para no sucumbir a la soledad, a los múltiples obstáculos del escenario familiar y a los insistentes estímulos de distracción del escenario virtual. Por ello, tanto el contenido como las formas y procedimientos deben implicar un plus de interés y relevancia de las propuestas pedagógicas (vídeos, juegos, indagaciones, diseño y producción, simulaciones, juegos de rol, trabajo cooperativo, presentaciones e intercambios, laboratorios y museos virtuales...). La presencia como disponibilidad, cuidado y atención de los docentes y de los pares es más imprescindible si cabe en el escenario virtual. El trabajo en grupo en torno a proyectos comunes se manifiesta como una de las estrategias más prometedoras y necesarias para abordar el aislamiento. En consecuencia, los canales, las plataformas y las herramientas digitales deben enriquecerse para permitir de manera ágil y efectiva el intercambio, la comunicación, la expresión y la relación a distancia, prestando especial cuidado a la gestión de los tiempos de exposición a las pantallas, para evitar la saturación insana, en virtud de las diferentes edades de los aprendices.

\subsection{La evaluación como aprendizaje}

Esta nueva cultura pedagógica exige una manera de entender la evaluación coherente y convergente con los principios que hemos explicitado con anterioridad. Tal vez sea en la manera de concebir y desarrollar la evaluación, reducida a la calificación de aprendizajes superficiales y reproducción de informaciones, donde la escuela convencional requiere un proceso de mayor y más sustantiva transformación de sus marcos pedagógicos. Una enseñanza basada en la libertad y responsabilidad, de riesgo y cuidado mutuo, de vulnerabilidad y creación exige el correlato de un feedback sincero, constructivo y comprometido, que ayude y estimule a aprender en colaboración, que inspire el crecimiento y el compromiso. Dar y pedir feedback es una forma de aprender y crecer, de entender nuestra vulnerabilidad constitutiva, de fortalecer la alianza entre aprendices, sin miedo al error, al ridículo y a la vergüenza que habitualmente atenaza y secuestra las posibilidades insospechadas de aprender en colaboración (Brown, 2016).

Coherente con los propósitos de esta nueva cultura pedagógica, se impone ser consecuentes y honestos con la clásica y necesaria diferencia entre evaluación y calificación. La evaluación educativa, formativa o auténtica, puede entenderse como un proceso complejo de indagación y diagnóstico continuado y compartido, que ayude al aprendiz a conocer sus fortalezas y debilidades, así como su génesis, a reflexionar sobre el valor de sus adquisiciones y asumir la regulación de su propio proceso de aprendizaje. La calificación es, por el contrario, un mecanismo que reduce el diagnóstico a un número, una nota, que etiqueta y clasifica pero que no ayuda a comprender la situación interna y externa en que se encuentra el aprendiz y mucho menos a rastrear las causas o factores que pueden explicar su historia y su génesis.

La evaluación educativa, entendida como feedback sincero, constructivo y comprometido es un componente sustantivo en el proceso de aprendizaje profundo y relevante. Debe tener bien claro el horizonte de la coevaluación y la autoevaluación, de modo que, progresivamente, sea el propio sujeto y el grupo de aprendices los que aprendan a observar, analizar y reflexionar sobre la calidad y naturaleza de sus propias prácticas, de sus propios proyectos académicos, personales y profesionales.

Este tipo de evaluación formativa, educativa o autentica, requiere la utilización de procedimientos sensibles a la complejidad, la interacción, la historia, los elementos más cualitativos de la vida humana y por tanto la construcción compartida de instrumentos plurales y flexibles con la mayor sensibilidad a 
las interacciones cognitivas y socioemocionales. El portafolios, la observación y registro de la práctica, la documentación pedagógica, el dialogo, el debate, la tutorización cercana, la elaboración de diarios, la construcción de historias de vida, los grupos focales, los informes y análisis compartidos, son ejemplos de los múltiples procedimientos que pueden utilizar docentes y aprendices en cada situación singular.

En la escuela convencional existe una hipertrofia de calificación, o evaluación sumativa y una atrofia preocupante de la evaluación educativa, tendencia que hay que invertir si queremos ayudar al desarrollo de la sabiduría. El criterio genérico podría expresarse en la siguiente recomendación: más evaluación educativa y menos calificación. Sería recomendable no utilizar la calificación individual y sancionadora en las etapas obligatorias del sistema educativo, puesto que no hay ninguna necesidad de clasificar, ni seleccionar cuando nos proponemos potenciar el desarrollo más satisfactorio de la personalidad de cada aprendiz hasta el máximo de sus posibilidades. Cuando, como por ejemplo en la universidad, se justifica la calificación individual para facilitar la selección y acreditación más justa de los futuros profesionales, esta debería ser el resultado de largos, complejos, transparentes y completos procesos de evaluación formativa holística y basada no solo en los conocimientos declarativos sino en el pensamiento práctico, crítico y creativo que debe manifestarse en las actuaciones, en los diagnósticos, diseños, proyectos, desarrollos y valoraciones que exhiben los universitarios cuando se implican en los problemas auténticos de su quehacer profesional en los contextos reales de su práctica.

\section{La investigación acción cooperativa (Lesson Study) como piedra angular de una nueva cultura pedagógica}

Como no parece muy coherente predicar lo que no se práctica, nos gustaría concluir esta reflexión sobre la innovación en metodología y evaluación educativa con una propuesta pedagógica que venimos desarrollando en los últimos 10 años en la Universidad de Málaga: Lesson Study (LS) como proceso de mejora de la enseñanza, evaluación y aprendizaje y sobre todo de reconstrucción del conocimiento práctico de los docentes.

La LS es una estrategia centenaria originaria de Japón con herencia europea ${ }^{9}$ que incorpora un proceso cooperativo de investigación/acción de progreso en espiral. Puede considerarse una estrategia privilegiada para el desarrollo de la improvisación disciplinada, reflexiva y cooperativa, que hemos explorado y experimentado tanto en la formación inicial como en la formación permanente del profesorado, pero igualmente relevante, en nuestra opinión, para otras etapas y otros dominios del saber y del quehacer académico.

\subsection{La LS en la Universidad de Málaga}

Desde 2009, la LS nos ha inspirado y ayudado a definir las cualidades pedagógicas del proceso formativo de los docentes en la Facultad de Educación de la Universidad de Málaga. Un proceso lento, sostenido y compartido que ha ido creciendo.

Inicialmente, la LS nos ayudó a mejorar y a transformar nuestra propia práctica como docentes universitarios en el contexto del Máster de Políticas y Prácticas de Innovación Educativa. Una experiencia que nos animó a iniciar diferentes investigaciones, con maestras y maestros de educación infantil y a introducirlo en la formación inicial.

En el curso 2013/14, incorporamos la LS en el Practicum III y el TFG del Grado de educación Infantil (Soto et al., 2016). La normativa del TFG de nuestra Facultad generó un marco idóneo para vincular la investigación con la práctica, a través de procesos de acción reflexiva. Con la LS incorporamos el decisivo componente cooperativo, rompiendo es aislamiento tradicional de los aprendices en el practicum. Las estudiantes tutorizadas en grupos de 4-6, investigan, diseñan, desarrollan, analizan y reformulan sus propuestas didácticas en los diferentes contextos de prácticas, generalmente, con la complicidad de las tutoras profesionales.

9 Puede encontrarse más información en las siguientes publicaciones Pérez Gómez y Soto (2011); Soto y otros (2015, 2019). 
Este proceso cuajado de análisis, diarios, retroalimentaciones y debates continuos entre la realidad del aula, sus experiencias docentes y las recién estrenadas teorías, fortalece y facilita la reconstrucción de su conocimiento práctico en compañía. Los informes correspondientes se muestran y comparten en un contraste ampliado al presentar sus Trabajos de Fin de Grado ante un tribunal externo que, sorprendido, atribuyó, inicialmente, a las capacidades singulares de las estudiantes y no al proceso, la calidad y profundidad de las reflexiones y documentaciones de las propuestas defendidas. El conocimiento más complejo se ha encarnado en sus prácticas y éstas ofrecen un entramado didáctico y organizativo coherente con ellas. Así fue como algunos compañeros y compañeras de la Universidad empezaron a conocer la Lesson Study.

Sin duda, la potencialidad de la experiencia nos animó a extenderla más allá del practicum. Incorporamos las LS a un proyecto coordinado e interdisciplinar del Primer curso del Grado de Infantil que incluía las asignaturas de Didáctica General, Organización Escolar y Análisis de la práctica en una experiencia de aprendizaje y servicio. Los estudiantes tendrían que diseñar, desarrollar, evaluar y reformular una propuesta didáctica, organizativa e inclusiva para niños y niñas de infantil en sus centros escolares y en la propia Facultad. Lo resultados han sido, por lo general, notablemente satisfactorios (Soto et al., 2019)

Seis principios han sostenido estos programas de investigación acción cooperativa:

La relevancia e interdisciplinariedad del conocimiento: aprender con sentido. Encontrar espacios para vincular la teoría y la práctica, requería esencializar e integrar el currículum de diferentes asignaturas para favorecer la emergencia, el cuestionamiento, el contraste y la reconstrucción de su conocimiento pedagógico práctico. Se sustituye un currículum organizado en disciplinas por un currículum basado en problemas, casos y proyectos.

La cooperación y experimentación como eje metodológico: Lesson Study. El alumnado en grupo de 5-6, siguen las fases de la Lesson Study ${ }^{10}$ para repensar y recrear su experiencia previa, analizando la virtualidad pedagógica de la misma. La LS puede considerarse una experiencia de alianza de aprendices que comparten una intención común y se atreven a explorar el incierto proceso de experimentación pedagógica, intercambiando y contrastando pensamientos, propuestas y acciones, en un clima de confianza, apoyo y estimulo mutuo.

Aprender con responsabilidad y compromiso: Aprendizaje y Servicio. Al responder a necesidades reales del entorno, se favorece el desarrollo de la sensibilidad y compromiso social, un aprendizaje contextualizado y un servicio mutuo que apela directamente a las disposiciones de nuestros estudiantes (actitudes, emociones y valores), generalmente ausentes de los objetivos de aprendizaje universitario y que son tan necesarios para tejer y desarrollar sus competencias profesionales.

La evaluación formativa: el Portafolio Educativo. Para regular todo este proceso, es necesario buscar una estrategia de evaluación, autoevaluación y coevaluación, educativa o auténtica que favorezca un proceso de aprendizaje más autónomo. El portafolio digital, a través del diario y otras producciones compartidas, hace posible que el alumnado incorpore la documentación y la reflexión cotidiana como hábitos, estimulando la expresión creativa y crítica a través de la palabra escrita, la imagen o el vídeo.

La tutorización como estrategia: La enseñanza personalizada. El tamaño de los grupos en las Facultades de Educación sabotea las mejores intenciones docentes. El Espacio Europeo de educación Superior incorporó algunos cambios sugerentes, pero su desarrollo en la universidad española olvidó generar las estructuras y condiciones para que el cambio fuera posible. Para facilitar la personalización de la enseñanza, desarrollamos una tutoría interdisciplinar, distribuyendo los estudiantes entre los docentes implicados. Esta estructura permitió desarrollar un espacio de mentoría cercano, delicado y continuo de reflexión dialogada y de contraste de ideas (Muñoz González et al.,, 2019), ya que cuestionar las ideas y valores, que definen la propia identidad, puede suponer un proceso de alta tensión emocional en el aprendiz de docente, precisamente cuando su status no le proporciona las mejores condiciones para cuestionar las ideas, hábitos, creencias y valores, ni para desnudar sus incertidumbres y deficiencias.

$101^{\text {a }}$ Fase: Definición del problema. 2a Fase: Diseño cooperativo de una lección experimental. $3^{a}$ Fase: Enseñar y observar la lección. $4^{\text {a }}$ Fase: Discusión y recogida de evidencias. $5^{\text {a }}$ Fase: Revisión de la lección experimental. $6^{\text {a Fase: }}$ Desarrollo de la lección experimental con otro grupo. $7^{\text {a }}$ Fase: Discusión sobre las nuevas evidencias y difusión de la experiencia. 
Una nueva organización del tiempo y del espacio. Crear escenarios auténticos requiere desbordar, abrir y diversificar los espacios y los tiempos de aprendizaje en la Universidad y conectarlos con escenarios escolares estimulantes. La estrecha relación con las escuelas ha sido una constante de nuestra manera de entender la formación inicial más allá del practicum.

A partir del año 2018, la LS se extendió a través de un segundo Proyecto de investigación financiado ${ }^{11}$ al Grado de Primaria y al Grado de Pedagogía, a través de 10 estudios de casos que nos han ayudado a comprender la diversidad de posibilidades y obstáculos en los diferentes escenarios y carreras de la formación inicial. La LS, el portafolios y la tutorización, incorporados en contextos de sentido, se consolidan como herramientas pedagógicas coherentes con los principios que hemos ido desgranando a lo largo de este escrito.

En primer lugar, porque la alianza de aprendices que genera el proceso de LS rompe el aislamiento secular de los docentes en todas las fases del desarrollo de su práctica: diagnóstico, diseño, planificación, ejecución y evaluación. Este crecer en relación arropa a cada agente implicado en el complejo viaje de aceptar su consustancial vulnerabilidad, la provisionalidad de su saber hacia la transformación de sus creencias, actitudes y hábitos, extraordinariamente resistentes al cambio, y, sobre todo, proporciona el coraje y la seguridad para sumergirse en experiencias de riesgo por la novedad y complejidad de las propuestas pedagógicas que se han atrevido a experimentar.

En segundo lugar, porque estimula la transformación del pensamiento práctico como eje de construcción de la sabiduría pedagógica de los docentes a través de la experimentación curricular concreta, creativa y reflexiva. La personalización cooperativa de la enseñanza ha provocado una experiencia totalmente nueva en la vida académica de nuestras estudiantes, y, sobre todo, ha provocado la necesidad de reflexionar e investigar sobre su propia práctica.

Esta experiencia junto con el Practicum han sido las experiencias más auténticas y reveladoras que he tenido en mi formación en la Facultad. (Entrevista a una estudiante del Grado de Infantil dos años después de su implicación en la LS)

Sin embargo, también nuestra experiencia muestra la complejidad de desarrollar proyectos de carácter interdisciplinar, innovador y coordinado en el contexto universitario. Poner en marcha y sostener una práctica docente como la descrita no está exenta de dificultades y retos. Desbordar las rígidas estructuras universitarias, conectar con las escuelas, transformar el tiempo y el espacio, buscar los tiempos para la coordinación, e incluso la tarea de persuasión inicial con las estudiantes, acostumbradas a otros modos de aprender y sobre todo de evaluar (calificar), se tornan a veces, en dilemas de sostenibilidad para la propia experiencia.

La Universidad ha de repensar no solo qué necesita saber un maestro o maestra sino también, el cómo debe aprenderlo. Generar contextos de acción y reflexión cooperativa que les ayude a aprender a pensar, hacer y sentir como docentes. A pesar de las dificultades para encajarlo en el andamiaje universitario, testimonios como los de esta estudiante siguen estimulando nuestra tarea:

Gracias a la Lesson Study he aprendido un montón de cosas, pero ya no sólo como docente, sino como persona. Y eso, que lo consigan unas profesoras de la facultad, me parece una utopía. Para mí es increíble, que hayáis hecho eso en mí. (...). Este cuatrimestre ha sido como un cambio de todo, y yo creo que la mayoría por no decir todas, hemos cambiado muchísimo. Y me gustaría que todo eso que tenemos ahora no se pierda por el camino (...). Porque para mí esto no era venir a clase... Mis compañeras de piso decían: "pff, tengo que ir a clase" Yyo: "¡bien, tengo que ir a clase”! (...). ¿Pero no hacéis exámenes?”. "No, pero me llevo experiencias". (Amaia, evaluación final del proyecto; 6 de junio de 2019)

11 EDU2017-86082-P: “Lesson Studies. Escuela y universidad: Investigando la reconstrucción del conocimiento práctico en la formación inicial del profesorado” 


\section{Referencias}

Allen, J. Rowan, L. y Singh, P. (2020). Teaching and teacher education in the time of COVID-19. Asia-Pacific Journal of Teacher Education, 48(3), 233-236. https://doi.org/10.1080/1359866X.2020.1752051

Attali, J. (2020, 20 de julio). Coronavirus. Jacques Attali: La humanidad aún no comprendió la profundidad de la crisis que se avecina y el costo de la resurrección. La Nación. https://www.lanacion.com.ar/el-mundo/coronavirus-jacques-attalila-humanidad-aun-no-comprendio-nid

Barad, K. (2007). Meeting the universe halfway: Quantum physics and the entanglement of matter and meaning. Duke University Press.

Belavi, G. y Murillo, F. J. (2020). Democracia y justicia social en las escuelas: Dimensiones para pensar y mejorar la práctica educativa. REICE. Revista Iberoamericana sobre Calidad, Eficacia Cambio en Educación, 18(3), 5-28. https://doi.org/10.15366/reice2020.18.3.001

Belmonte, O., Cuesta, C., Guadix, N., Hernández, A., y López de Turiso, A. (2020). COVID-19 Reimaginar la educación. Aprendizajes de la pandemia sobre los que construir un pacto por la educación. UNICEF.

Brady, M. (2020, 14 de mayo). Why not reform education from the bottom up for a real change? Blog de Diane Ravitch. https://dianeravitch.net/2020/05/14/marion-brady-why-not-reform-education-from-the-bottom-up-for-areal-change/

Brantmeier, E. J. y Mackenna, M. K. (2020). Pedagogy of vulnerability. IAP-Information Age Publishing.

Brown, B. (2016). El poder de ser vulnerable: ¿Qué te atreverías a hacer si el miedo no te paralizara? Urano.

Escolar, I. (2020, 16 de julio). En defensa de lo público. ElDiario.es. https://www.eldiario.es/escolar/defensa-publico_132_6109474.html

Challenger, M. (2021). El animal que somos. Roca Editorial.

Christakis, N. (2019). Blueprint: The evolutionary origins of a good society. Little Brown Spark.

Claxton, G. y Lucas, B. (2015). Educating Ruby: What our children really need to learn. Crown House Publishing.

Barrett, L. (2018). La vida secreta del cerebro. Como se construyen las emociones. Paidós

Eagleman, D. (2020). The safety net. Canongate books.

Fuster, J. (2020). El telar mágico de la mente. Ariel.

Garcés. M. (2020) Escuela de aprendices. Galaxia Guttemberg.

Garret, L. (2020). The coming plague: Newly emerging diseases in a world out of balance. Amazon.

Gewertz C. (2020, 3 de junio). How technology, coronavirus will change teaching by 2025. Edweek.org. https://www.edweek.org/technology/how-technology-coronavirus-will-change-teaching-by-2025/2020/06

Harari, N. (2018). 21 lecciones para el siglo XXI. Debate.

Harari, N. (2020, 20 de marzo). The world after coronavirus. Financial Times. https://www.ft.com/content/19d90308-6858-11ea-a3c9-1fe6fedcca75

Hu, M., Arnesen, K., Barbour, M. K., Leeary, H. (2019). A newcomer's lens: A look at K-12 online and blended learning. Journal of Online Learning Research, 5(2), 123-144.

Innerarity, D. (2020). Una teoría de la democracia compleja: Gobernar en el siglo XX. Galaxia Guttemberg.

Kahneman, D. (2015). Pensar rápido pensar lento. Debate.

Kaiser, B. (2019). La dictadura de los datos. Harpercollins.

Konen, J. K. J. (2020). COVID-19 reality: 5 reasons why teachers are working harder now than they ever have. https://www.teacher.org/daily/covid-19-reality-5-reasons-why-teachers-are-working-harder-now-thanthey-ever-have 
Korthagen, F. (2017). A foundation for effective teacher education: Teacher education pedagogy based on theories of situated learning. En D. J. Clandinin y J. Husu (Eds.), The SAGE handbook of research on teacher education (pp. 528-544). Sage.

Louden, J. (2020) Why bother? Discover the desire for what's next. Page Two.

Luengo, F. y Manso, J. (2020). Informe de investigación COVID-19: Voces de docentes y familias. Fundación Atlántida.

Mitra, S. (2019). The school in the cloud: The emerging future of learning. Corwin.

Morín, E. (2020). Cambiemos de vía: Lecciones de la pandemia. Paidós.

Muñoz González, L., Serván, M. J. y Soto, E. (2019). Las competencias docentes y el portafolio digital: Crear espacios de aprendizaje y evaluación en la formación inicial del profesorado. Un estudio de casos. Revista Iberoamericana de Evaluación Educativa, 12(2), 111-131. https://doi.org/10.15366/riee2019.12.2

Noddings, N. (2018). Philosophy of education. Routledge.

O’Neil, C. (2018). Armas de destrucción matemática. Capitán Swing.

Pérez Gómez, A. I. (2012). Educarse en la era digital. Morata.

Pérez Gómez, A. I. (2017). Pedagogías para tiempos de perplejidad. Homo Sapiens.

Pérez Gómez, A. I. y Soto, E. (2011). Lesson study: La mejora de la práctica y la investigación docente. Cuadernos de Pedagogía, 417, 64-67.

Poyton, R. (2020). Pausa: No eres una lista de tareas pendientes. Koan Libros.

Pozo, I. (2020). La educación está desnuda. SM.

Pugh, G. (2019). Psychotherapy meets emotional neuroscience: The two minds of cognition and feeling. Routledge.

Ramonet. I. (2020, 25 de abril). La pandemia y el sistema-mundo. Le Monde Diplomatique. https://mondiplo.com/la-pandemia-y-el-sistema-mundo

Reimers, F. y Schleicher, A. (2020). Un marco para guiar una respuesta educativa a la pandemia de 2020 de la COVID-19. OEI.

Rivas, A. (2020). Quien controla el futuro de la educación? Siglo XXI.

Sangrá, A. (2020). Decálogo para la mejora de la docencia online propuestas para educar en contextos presenciales discontinuos. UOC.

Sawyer, K. (2017). Group genius. The creative power of collaboration. Basis Books.

Sawyer, K. (2019). The creative classroom. Innovative teaching for $21^{\text {st }}$ century learners. Teacher College Press.

Sennet. R. (2018). La cultura del nuevo capitalismo. Anagrama.

Seymur, R. (2020). The twittering machine. Akal.

Siegel, D. (2017). Viaje al centro de la mente. Paidos

Soloway, E.y Norris, C. (2020,12 de julio). How technology, coronavirus will change teaching by 2025. Education week. https://www.edweek.org/technology/how-technology-coronavirus-will-change-teaching-by-2025/2020/06

Soto, E., Pérez Gómez, A. I. y Rodríguez, C. (2019) Aprender a enseñar en la universidad. De la investigación acción a la Lesson Study. En N. de Alba Fernández y R. Porlan (Coords.), Docentes universitarios: Una formación centrada en la práctica (pp. 55-82). Morata.

Soto, E. Pérez Gómez, A. I., Serván, M. J. y Peña, N. (2015) Lesson study and the development of teacher's competences: From practical knowledge to practical thinking. International Journal for Lesson and Learning Studies, 4(3), 209-223. https://doi.org/10.1108/IJLLS-09-2014-0034

Soto, E., Pérez Gómez, A. I., Serván, M. J. y Peña, N. (2019) Action research through lesson study for the reconstruction of teachers' practical knowledge. A review of research at Málaga University (Spain). Educational Action Research, 27(4), 527-542. https://doi.org/10.1080/09650792.2019.1610020 
Soto, E., Serván, M. J. y Caparrós, R. M. (2016). Learning to teach with lesson study. The practicum and the degree essay as the scenario for reflective and cooperative creation. International Journal for Lesson and Learning Studies, 5(2), 116-129. https://doi.org/10.1108/IJLLS-12-2015-0042

Sternberg, R., Linda, J. y Grigorenko, L. (2009). Teaching for wisdom, intelligence, creativity and success. Corvin Press.

Torralba, F. (2020). Vivir en lo esencial: Ideas y preguntas después de la pandemia. Plataforma Editorial.

Trujillo-Sáez, F. (2020) Aprendery enseñar en tiempos de confinamiento: Propuestas útiles para la educación del siglo XXI en tiempos de pandemia. Libros de la Catarata.

Veliz, C. (2020). Privacy is power: Why and how you should take back control of your data. Transworld.

Vidal, H. E. (2020). El regreso triunfal de los oligopolios. Contexto y Acción, 262. https://ctxt.es/es/20200701/Firmas/32856/oligopolios-desregulacion-Glass-Steagall-robert-borkneoliberalismo-ernesto-h-vidal.htm .

Vidal, M. (2018). Elon Musk, Netflix, Microsofty el fin de la humanidad. https://www.marcvidal.net/blog/2018/3/16/elon-musk-netflix-microsoft-y-el-fin-de-la-humanidad

Willson, E. (2018). Los orígenes de la creatividad humana. Crítica.

Zhang, W. Wang, Y., Yang, L. y Wang, C. (2020). Suspending classes without stopping learning: China's education emergency management policy in the covid-19 outbreak. Journal of Risk and Financial Management, 13(3), 55 - 63. https://doi.org/10.3390/jrfm13030055

Zuboff, S. (2020). La era del capitalismo de la vigilancia: La lucha por un futuro humano frente a las nuevas fronteras del poder. Paidós.

\section{Breve CV de los/as autores/as}

\section{Ángel I. Pérez Gómez}

Catedrático Emérito del Dpto. de Didáctica y Organización Escolar de la Universidad de Málaga (UMA). Profesor en la Universidad de Salamanca, Complutense de Madrid, la Laguna y há sido Vicerrector en la UMA y en la UNIA y Gestor Nacional de Investigación. Ha recibido varios premios y condecoraciones: la Encomienda Alfonso X el Sabio al mérito docente y la medalla de oro del Ateneo de Málaga entre otros. Participa en el consejo asesor de varias revistas nacionales e internacionales. Ha escrito más de 20 libros y 150 artículos. Sus libros más recientes incluyen Pedagogías para tiempos de perplejidad (2017) Educarse en la era digital (2012). La cultura escolar en la sociedad neoliberal (1998). Actualmente trabaja en Investigación acción cooperativa (Lesson Study) y la reconstrucción del pensamiento práctico del professorado. Email: apgomez@uma.es

ORCID ID: https://orcid.org/0000-0001-8291-0849

\section{Encarnación Soto Gómez}

Profesora Titular del Dpto. de Didáctica y Organización Escolar de la Universidad de Málaga y Coordinadora del Máster de Innovación Educativa. Investigadora y responsable de proyectos relacionados con la innovación, la evaluación y la formación docente a través de las Lesson Study. Entre las últimas publicaciones destacan: "Lesson study and the development of teacher's competences: from practical knowledge to practical thinking” (IJJLS, 2015). El monográfico: "Lesson Study, investigación acción cooperativa para formar docentes y recrear el curriculum” (RIFOP, 2015). "Aprender a enseñar en la universidad: de la investigación acción a las Lesson Study” en Docentes Universitarios. Una formación centrada en la práctica (Morata, 2019) Action research through lesson study for the reconstruction of teachers' practical knowledge. EAR, 2019).Email: esoto@uma.es

ORCID ID: https://orcid.org/0000-0001-5758-1684 